\title{
A Complete Model of the Signal in Surface Detector Arrays and its Application for the Reconstruction of Mass-sensitive Observables
}

\author{
Maximilian Stadelmaier, ${ }^{*}$ Markus Roth, David Schmidt and Darko Veberič \\ Institute for Astroparticle Physics, Karlsruhe Institute of Technology $\mathbf{Q} \mathbf{I T}$, Germany \\ E-mail: max.stadelmaier@kit.edu
}

The principle of air-shower universality yields a method of understanding extensive air showers of UHECRs as a superposition of different particle components whose spatial and temporal distributions follow individual analytical profile functions. We present a model of the expected densities of particles in time and space that uses the depth of the shower maximum, $X_{\max }$, and the relative muonic content of the shower, $R_{\mu}$, as input parameters besides the shower geometry and energy. The model is parametrized using simulated showers using different hadronic interaction models. Furthermore, we present results for the reconstruction of $X_{\max }$ and $R_{\mu}$ that allow for an event-by-event estimation of the mass of the primary particle, based on the responses of the water-Cherenkov and scintillator surface detectors of the Pierre Auger Observatory.

$3^{\text {th }}$ International Cosmic Ray Conference (ICRC 2021)

July 12 th - 23rd, 2021

Online - Berlin, Germany

\footnotetext{
${ }^{*}$ Presenter
} 


\section{Introduction}

Ultra-High Energy Cosmic Rays (UHECRs, above $10^{19} \mathrm{eV}$ ), being the highest energetic particles known to mankind, challenge the theoretical limits for their sheer existence [1]. Even though experimental evidence implies that the spectrum of cosmic rays is heavily dominated by ionized nuclei for energies above $10^{18} \mathrm{eV}$ [2], the exact chemical composition of the UHECRs is still unknown.

Due to the increasingly lower flux at the highest energies, UHECRs are not observed directly, but by the Extensive Air Shower (EAS) that is the cascade of secondary particles that is created when an UHECR reaches the atmosphere of the earth. For the highest energies, the footprint of an EAS at ground level reaches several kilometers in diameter and can therefore be detected by an array of surface detectors [3].

To estimate the chemical composition of the UHECRs that produce EASs, the precise understanding of this phenomenon is crucial. Especially the depth of the maximum calorimetric energy deposit in the atmosphere, $X_{\max }$, and the relative amount of muons in the EAS, $R_{\mu}$, are strong indicators for a certain primary mass [4], as studied by [5, 6]. In this work we present an analytical model of the expected signal in surface detector arrays and its dependency on $X_{\max }$ and $R_{\mu}$ that is based upon the concept of air-shower universality.

\section{Extensive air showers}

The impact of an UHECR on Earth's atmosphere creates an EAS which is a cascade of secondary particles that reaches out several kilometers in depth and width. Most particles are created in the core of the EAS, which coincides with the continued trajectory of the UHECR and which defines the shower axis as a line of reference. The plane that lies perpendicularly to the shower axis and that contains the shower core is defined as the shower plane.

Even though the particle content of the EAS is mostly defined by the hadronization that occurs in the first interactions, most of the particles in the overall cascade are created by electromagnetic processes. Most of the energy is therefore deposited in the atmosphere by electrons, positrons and photons. Muons, which are created mostly from the decay of pions, take a crucial role in the observation of UHECRs. Firstly they provide a measure for hadronization that took place in the first interactions of the shower and secondly they are the most long-lived visible particles of the EAS and therefore prominently detected by surface detectors. Since the hadronization of the first interactions is enhanced by the number of nucleons taking part in the first interaction, the relative amount of muons in an EAS is an indicator of the mass of the primary particle, see e.g. [7].

\section{Universality of extensive air showers}

The concept of air-shower universality as first studied by [8] and employed by $[9,10]$ states that the fractional rate of change in the calorimetric energy deposit with respect to shower age is approximately the same for electromagnetic showers from UHECRs. The shower age is a quantity defined relative to the atmospheric depth of the maximum energy deposit in the atmosphere, $X_{\max }$, and is usually described by $s=3 X /\left(X+2 X_{\max }\right)$, where $X$ is the slant depth of the shower core 
measured from the top of the atmosphere along the shower axis. The calorimetric energy deposit along the shower axis can be described by a longitudinal profile function, as found by [11]. Further, the mean angular and lateral distributions of the electromagnetic particles in extensive air showers as well as their energy spectra can be universally parametrized by the total number of particles and the shower age $[12,13]$. This implies that the expected density of particles of the electromagnetic cascade at any given point in the air shower can be given as function of the shower age using a longitudinal and a lateral profile function. Further, the concept of universality has been extended to describe showers from hadronic UHECRs [14-16]. In this context, asides from the electromagnetic component of an EAS, three particle components were identified, which are each described by an individual longitudinal and lateral profile and for which the concept of universality holds with reasonable approximation, such that air-shower universality is achieved also for EAS from hadronic UHECRs.

\subsection{The four-component Heitler-Matthews model}

In the context of this work, we consider an EAS to be made up of the four particle components introduced by [15]. The electromagnetic component, e $\gamma$, contains electrons and positrons, as well as photons created by the electromagnetic cascade of the air shower. The muonic component, $\mu$, contains all muons and antimuons of the EAS. The electromagnetic component from muon decay, e $\gamma(\mu)$, contains electromagnetic particles that were created in the decay of particles from the muonic component in first or second generation. The hadronic component, e $\gamma(\pi)$, contains all electromagnetic particles that were created as a result from the decay of hadrons in first and second generation as well as all hadronic jets.

These four components do not scale independently of each other, and expressions for their expected correlation can be given according to the superposition model described in [17]. The total number of particles of a given component in an EAS, $N_{i}$, where $i \in\{\mathrm{e} \gamma, \mu, \mathrm{e} \gamma(\mu), \mathrm{e} \gamma(\pi)\}$, with respect to the average amount of respective particles in proton showers of the same energy, $\left\langle N_{i}^{\mathrm{p}}\right\rangle$, is given by

$$
N_{i}=R_{i}\left\langle N_{i}^{\mathrm{p}}\right\rangle .
$$

For convenience, the scale of the individual components is expressed as a function of $R_{\mu}$. In first order, all components scale linearly with respect to $R_{\mu}$ according to the relation

$$
R_{i}-1=\alpha_{i}\left(R_{\mu}-1\right)
$$

Since the number of particles in the electromagnetic component scales asymptotically independently of the hadronization of the first interactions, $\alpha_{\mathrm{e} \gamma}$ is expected to be very close to 0 , but slightly smaller than 0 due to energy conservation. For the latter two components we set, $\alpha_{\mathrm{e} \gamma(\mu)}=1$ and $\alpha_{\mathrm{e} \gamma(\pi)} \approx 1.2$ (possible negative values for $N_{\mathrm{e} \gamma(\pi)}$ are omitted).

Except for their correlation according to Eq. (2) the four components are treated individually and independently in the context of this work.

The energy dependency of the number of particles is given by a power-law, individually for the four components. As a reference, the expected number of particles produced by a proton shower of $E_{\text {prim }}=10^{19} \mathrm{eV}$, written $N_{i}^{19}$, is used for each component. The expected number of particles from 
an UHECR of energy $E$ is then given by

$$
N_{i}(E)=\left(\frac{E}{10^{19} \mathrm{eV}}\right)^{\gamma_{i}} N_{i}^{19} .
$$

While the exact numerical value for each $\gamma_{i}$ varies for different different hadronic interaction models, the best fit results obtained in the context of this work are $\gamma_{\mathrm{e} \gamma} \simeq 0.98, \gamma_{\mu} \simeq 0.96, \gamma_{\mathrm{e} \gamma(\mu)} \simeq 0.95$, and $\gamma_{\mathrm{e} \gamma(\pi)} \simeq 0.94$ for Epos-LHC. Slightly different values are obtained for SibYLL2.3C and QGSJETII-04.

\subsection{The longitudinal profile}

The Gaisser-Hillas profile function accurately describes the longitudinal development of EAS, as directly measured by [18]. To describe the expected particle density $\varrho(r, X)$ along a straight line parallel to the shower axis at distance $r$, we use a modified version of the Gaisser-Hillas profile. Firstly, instead of the slant depth with respect to the top of the atmosphere, $X$, the slant depth of the shower core with respect to the shower maximum, $\Delta X=X-X_{\max }$, is used because of the universal behavior of air showers in the region of $\Delta X \simeq 0$. Secondly, a reference depth $\Delta X_{\text {ref }}$ is introduced,

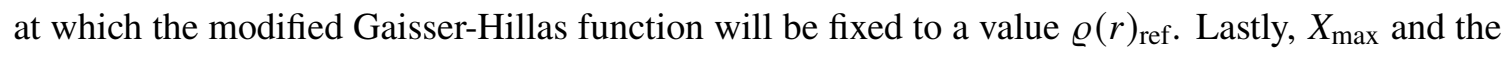
depth of the first interaction, $X_{1}$, are replaced by the respective quantities $\Delta X_{\max }$ and $\Delta X_{1}$, measured relatively to the depth $X_{\max }$ along the shower axis. The modified Gaisser-Hillas profile takes the form

$$
\varrho(r, \Delta X)=\varrho(r)_{\operatorname{ref}}\left(\frac{\Delta X-\Delta X_{1}}{\Delta X_{\max }-\Delta X_{1}}\right)^{\frac{\Delta X_{\mathrm{ref}}-\Delta X_{1}}{\lambda}} \mathrm{e}^{-\frac{\Delta X-\Delta X_{\mathrm{ref}}}{\lambda}} .
$$

The parameters $\Delta X_{\max }$ and $\lambda$ are obtained from simulations and are linearly dependent on the radial distance to the shower core, $r$, especially concerning the $\mu$ and e $\gamma(\mu)$ component. This is in agreement with [19] after which the shower maximum is geometrically retarded and the shower profile is stretched with increasing distance from the shower core.

\subsection{The lateral reference profile}

The lateral behaviour of the expected particle density at a fixed reference depth, $\varrho(r)_{\text {ref }}$, is described by the NKG function, which was derived by Greisen [4] as an approximation to the solutions to the cascade equations of Nishimura and Kamata [20]. Using $N$ as the number of particles expected in the shower from a single component as described in Eq. (3), the function takes the form

$$
\varrho(r)_{\mathrm{ref}}=N \frac{\Gamma(9 / 2-s)}{2 \pi r_{\mathrm{G}}^{2} \Gamma(s) \Gamma(9 / 2-2 s)}\left(\frac{r}{r_{\mathrm{G}}}\right)^{s-2}\left(1+\frac{r}{r_{\mathrm{G}}}\right)^{s-9 / 2} .
$$

Even though Eq. (5) describes the behaviour of the e $\gamma$ - and e $\gamma(\pi)$-component very well, some modification is needed to describe the slanting behaviour of the lateral muon distribution (as also found in [4]). Therefore the lateral profile for the $\mu$ - and e $\gamma(\mu)$-component is additionally multiplied by a sigmoid-like function, moderating Eq. (5) at large distances.

\subsection{Corrections of azimuthal asymmetry}

For showers with a zenith angle of $\theta \neq 0$, azimuthal asymmetry of the signal is expected in the ground coordinate system. At first order this asymmetry is well described by the difference in $\Delta X$. A second order correction, which is necessary due to the non-linear behaviour of the atmosphere, can be applied by the factor $c_{\psi}=\exp \left(c_{0} r \Delta X \cos \psi / \lambda\right)$, where $c_{0}=O\left(10^{-5} \mathrm{~m}^{-1}\right)$. 


\subsection{The temporal distribution of particles}

Given the simplified assumption that many particles propagate with the speed of light on approximately rectilinear trajectories from their point of origin in the shower core to the point of their detection, we deduce that particles arrive time-ordered with respect to their creation at a given detector. Therefore, assuming there are $n$ particles at a given depth $X$ of the shower core, $n^{\prime}$ corresponding particles arrive at a given time $t$ at a detector, fulfilling the relation $n \mathrm{~d} X \propto n^{\prime} \mathrm{d} t$. The temporal distribution of particles arriving at a detector is therefore connected to the longitudinal profile of the shower. The time at which particles originating in the shower maximum arrive at a given detector can therefore be estimated by calculating the quantile $q_{X_{\max }}$ of particles present at the shower maximum, relative to the integrated longitudinal profile of the shower, $n_{\text {tot }}$,

$$
q_{X_{\max }}=\frac{1}{n_{\mathrm{tot}}} \int_{0}^{X_{\max }} n(X) \mathrm{d} X=\frac{1}{n_{\mathrm{tot}}^{\prime}} \int_{t_{\mathrm{pf}}}^{t_{X_{\max }}} n^{\prime}(t) \mathrm{d} t,
$$

using the integrated number of particles at a given detector, $n_{\mathrm{tot}}^{\prime}$, and the time of the plane-front shower arriving said detector, $t_{\mathrm{pf}}$, corresponding to particles originating from an atmospheric depth of $X=0$ at infinite Euclidean distance. Using the Gaisser-Hillas profile to describe $n(X)$, we find that $q_{X_{\max }} \simeq 40 \%$ for reasonable values of $X_{\max }$ and $\lambda$, and therefore choose $t_{X_{\max }} \simeq t_{40}$. Relative to the time of the plane-front shower, $\Delta t_{j}:=t_{j}-t_{\mathrm{pf}}, \Delta t_{40}$ can be expressed in terms of the atmospheric scale height $h_{\mathrm{s}}$, the zenith angle of the cosmic ray, $\theta$, the speed of light, $c$, and the projected slant depth of a station relative to the shower maximum, $\Delta X$, yielding

$$
c \Delta t_{40}=\sqrt{\sec ^{2} \theta\left(h_{\mathrm{s}} \ln \left(1+\frac{\Delta X}{X_{\max }}\right)\right)^{2}+r^{2}}-\sec \theta h_{\mathrm{s}} \ln \left(1+\frac{\Delta X}{X_{\max }}\right) .
$$

To match the behaviour observed in simulation data a radially dependent correction $c t_{0}(r)$ and a zenith-dependent shift $\Delta X \rightarrow \Delta X+\delta X(\sin \theta)$ is employed for Eq. (7) in the later model.

The temporal distribution of particles from an EAS arriving at a surface detector is well described by a log-normal distribution [21], which can be parametrized by the start time of the signal, $t_{\mathrm{s}}$, the time span $\Delta t_{40}$, and the shape-parameter $\sigma$.

\section{Parametrization of the expected signal in a surface detector}

Using the assumptions, that the signal deposited in a surface detector, $S$, is proportional to the number of particles arriving at the respective detector individually for every particle component and that the total signal is given by the sum of the signal of the components, we write the total signal in a surface detector as

$$
S_{\mathrm{tot}}=\sum_{i} R_{i} S_{i}, \quad i \in\{\mathrm{e} \gamma, \mu, \mathrm{e} \gamma(\mu), \mathrm{e} \gamma(\pi)\}
$$

using $R_{i}$ as a function of $R_{\mu}$ as described in Eq. (2). The profile given by Eq. (4) is fit to simulations of the surface detector response using 1080 proton-induced showers, generated by CoRsIKA v7.5 [22] using EPOS-LHC [23]. For several fixed radii, best fit results for $\varrho(r)_{\text {ref., }} \Delta X_{\max }, \lambda$ and $\Delta X_{1}$ are obtained for each particle component. A linear fit is performed for the individual best-fit results of $\Delta X_{\max }$ and $\lambda$, fixing their radial dependency. The individual best-fit results for $\varrho(r)_{\text {ref }}$ are fit to 

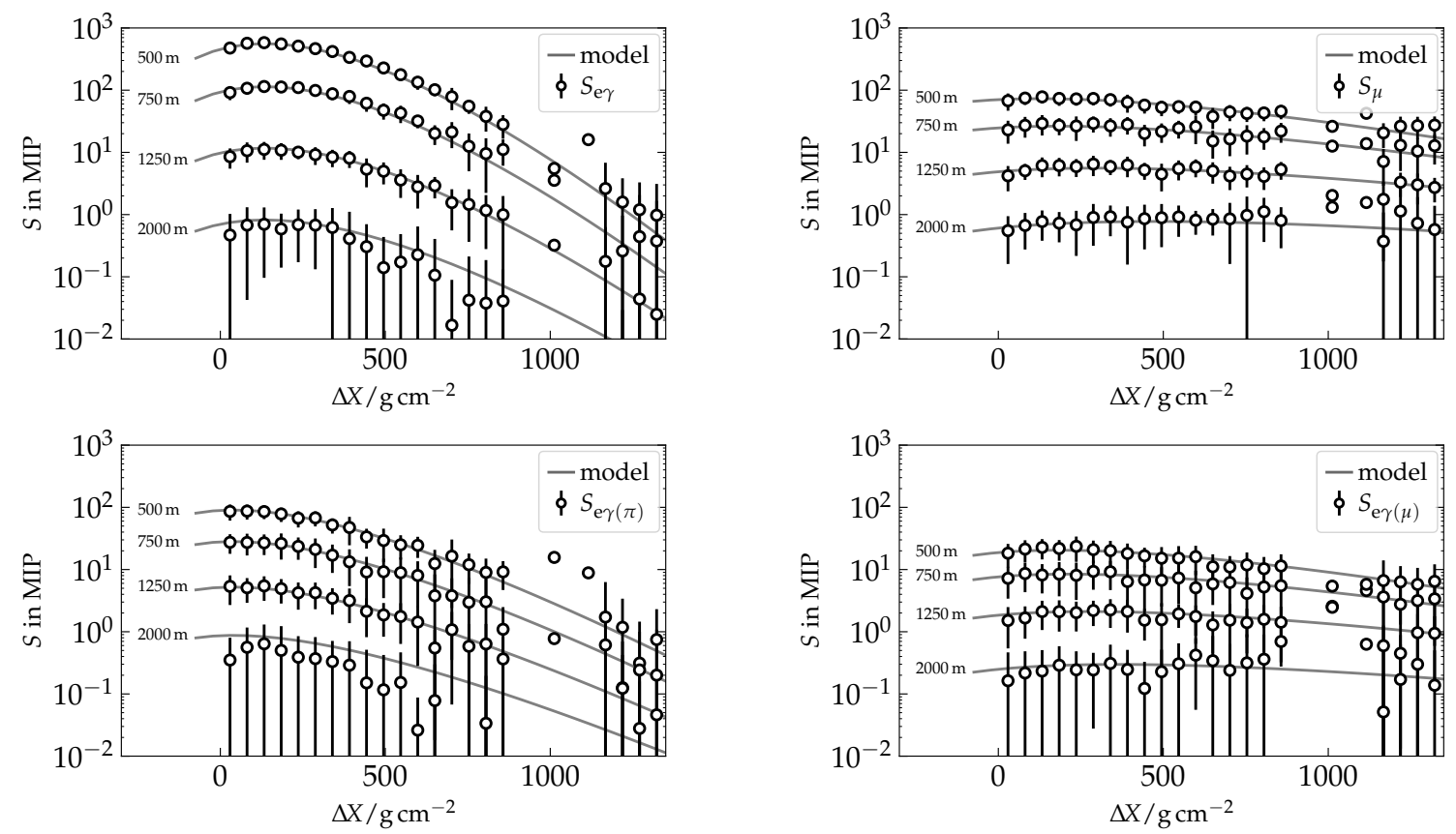

Figure 1: Simulated scintillator surface detector responses to EAS from protons of $10^{19} \mathrm{eV}$ with respect to the distance to the shower axis and $\Delta X$ and the corresponding model for the four particle components.

Eq. (5), fixing $N, s$ and $r_{\mathrm{G}}$ for each particle component, where $N$ is fit in terms of signal rather than particle count. The energy dependency is fixed by comparing best-fit results for $\varrho(r)_{\text {ref }}$ using simulated cosmic rays of different energies, assuming

$$
\varrho(r)_{\mathrm{ref}}=\left.\left(\frac{E}{10^{19} \mathrm{eV}}\right)^{\gamma} \varrho(r)_{\mathrm{ref}}\right|_{E=10^{19} \mathrm{eV}},
$$

according to Eq. (3), fixing $\gamma$ individually for each particle component. The total expected signal as from Eq. (8) treats $R_{\mu}$ and $X_{\max }$ as free parameters. The individual component signal is calculated according to

$$
\begin{aligned}
S_{i}= & N^{19}\left(\frac{E}{10^{19} \mathrm{eV}}\right)^{\gamma} \frac{\Gamma(9 / 2-s)}{2 \pi r_{\mathrm{G}}^{2} \Gamma(s) \Gamma(9 / 2-2 s)} c_{\psi} \\
& \left(\frac{r}{r_{\mathrm{G}}}\right)^{s-2}\left(1+\frac{r}{r_{\mathrm{G}}}\right)^{s-9 / 2}\left(\frac{\Delta X-\Delta X_{1}}{\Delta X_{\max }-\Delta X_{1}}\right)^{\frac{\Delta X_{\mathrm{ref}}-\Delta X_{1}}{\lambda}} \mathrm{e}^{-\frac{\Delta X-\Delta X_{\mathrm{ref}}}{\lambda}},
\end{aligned}
$$

where indices $i$ for the individual components were omitted on all parameters for better legibility. The agreement of the model for $S_{i}$ with simulated scintillator surface detector responses is depicted in Fig. 1.

The $40 \%$ time-quantile of the signal for stations of several different radii using showers of different zenith angles is fit to Eq. (7), fixing the corrections $c t_{0}(r)$ and $\delta X(\sin \theta)$ as polynomials in $r$ and $\sin \theta$, respectively. The speed of light as used in Eq. (7) is set to $\approx 95 \%$ of the speed of light in vacuum. The value of the shape parameter $\sigma$ is obtained from mean time and the standard deviation of the traces, which are both modelled to be linear in radius and $\Delta X$. The agreement of the model for $\Delta t_{40}$ and $\sigma$ with simulated data is depicted in Fig. 2. 

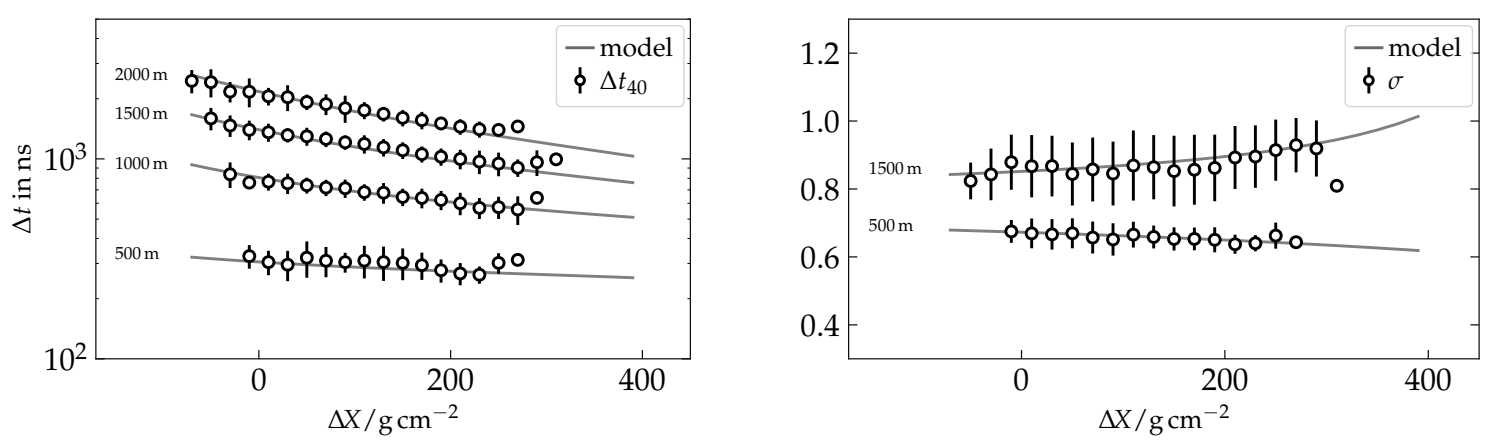

Figure 2: $\Delta t_{40}$ (left) and $\sigma$ (right) from the log-normal distribution fitted to simulated water-Cherenkov detector responses to EAS of UHECRs of $\theta=22^{\circ}$, with respect to the distance to the shower axis and $\Delta X$ as well as the corresponding model.

The cumulative time dependent signal is given by

$$
S\left(t>t_{\mathrm{s}}\right)=S_{\text {tot }}\left(\frac{1}{2}+\frac{1}{2} \operatorname{erf}\left(\frac{1}{\sqrt{2} \sigma} \ln \left(\frac{t-t_{\mathrm{s}}}{\Delta t_{40}-\Delta t_{\mathrm{s}}}\right)+\operatorname{erf}^{-1}(2 \times 0.4-1)\right)\right) .
$$

The parametrization is individually performed on simulated responses of water-Cherenkov and scintillator surface detectors of the Pierre Auger Observatory.

\section{The predictive power of the model}

Comparing the predicted signal response from the model parametrization to simulated data, a best-fit value for $R_{\mu}$ and $X_{\max }$ can be found on event level for EAS from cosmic rays of $E \gtrsim 10^{19} \mathrm{eV}$. Using a library of 16000 simulated showers of various energies and zenith angles, generated by CoRsiKa v7.5 using Epos-LHC, the quality of the reconstruction of $R_{\mu}$ and $X_{\max }$ was evaluated. The library contains EAS induced by Hydrogen, Helium, Oxygen and Iron nuclei. The precision and bias of $X_{\max }$ as well as $R_{\mu}$ are depicted in Fig. 3.

In a simplified approach, using the combined information of the reconstructed values for $R_{\mu}$ and $X_{\max }, \ln A$ of the primary particle can be estimated with a precision of ca. $\sigma(\ln A) \lesssim 1.5$ using principle component analysis on the data.

\section{Discussion and summary}

We present a full analytical model of the detector responses to particles from EAS based on the expected particle densities using implications from air-shower universality. We outline a way of parametrizing said model so that it can be applied on data to reconstruct $X_{\max }$ and $R_{\mu}$. The obtained precision for these observables is sufficient to reconstruct $\ln A$ with a sharp enough accuracy to discriminate between heavy and light primary particles on an event-by-event basis. 

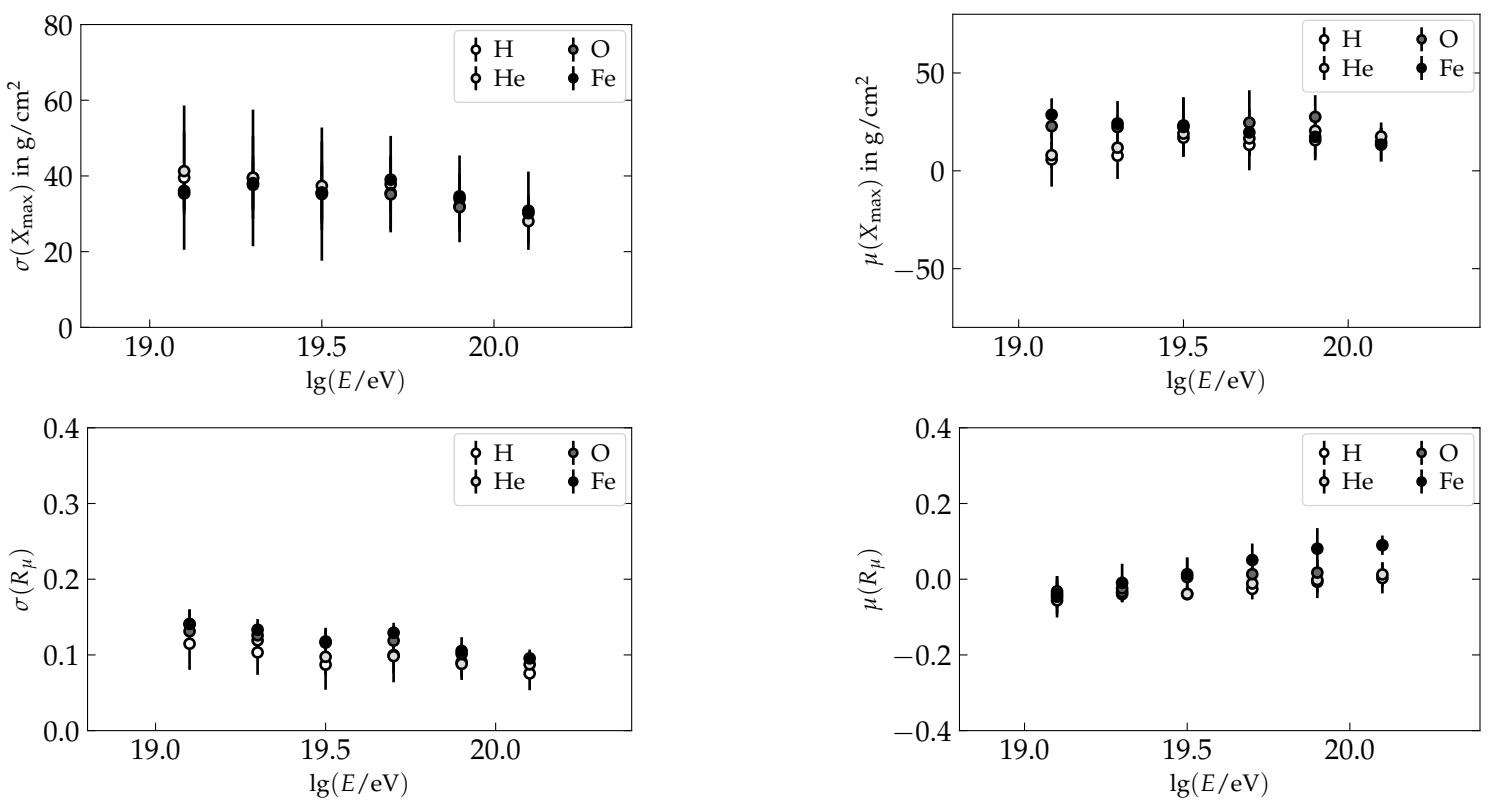

Figure 3: Precision $(\sigma)$ and bias $(\mu)$ for the reconstruction of $X_{\max }$ and $R_{\mu}$ with respect to the energy of the primary particle, for Hydrogen, Helium, Oxygen and Iron. Each data point shows mean and spread averaged over ten equidistant bins in zenith angle from $0^{\circ}$ to $50^{\circ}$.

\section{References}

[1] R. Alves Batista, J. Biteau, M. Bustamante, K. Dolag, R. Engel, K. Fang et al., Front. Astron. Space Sci. 6 (2019) 23.

[2] The Pierre Auger Collaboration, Phys. Rev. Lett. 125 (2020) 121106.

[3] The Pierre Auger Collaboration, Nuclear Inst. and Methods in Physics Research, A 798 (2015) 172.

[4] K. Greisen, Ann. Rev. Nucl. Sci. 10 (1960) 63.

[5] The Pierre Auger Collaboration, Phys. Rev. D 90 (2014) 122006 [1409. 5083].

[6] The Pierre Auger Collaboration, Phys. Rev. Lett. (2021) 152002 [2102 . 07797].

[7] The Pierre Auger Collaboration, Phys. Rev. Lett. 126 (2021) 152002.

[8] B. Rossi and K. Greisen, Rev. Mod. Phys. 13 (1941) 240.

[9] M. Giller, A. Kacperczyk, J. Malinowski, W. Tkaczyk and G. Wieczorek, J. Phys. G: Nucl. Part. Phys. 31 (2005) 947.

[10] P. Lipari, Nucl. Phys. B-Proc. Supp. 196 (2009) 309.

[11] T.K. Gaisser and A.M. Hillas, Proc. Int. Cosmic Ray Conf. 8 (1977) 353.

[12] S. Lafebre, R. Engel, H. Falcke, J. Hörandel, T. Huege, J. Kuijpers et al., Astropart. Phys. 31 (2009) 243.

[13] F. Nerling, J. Blümer, R. Engel and M. Risse, Astropart. Phys. 24 (2006) 421.

[14] M. Ave, R. Engel, J. Gonzalez, D. Heck, T. Pierog and M. Roth, Proc. Int. Cosmic Ray Conf. 2 (2011) 178.

[15] M. Ave, R. Engel, M. Roth and A. Schulz, Astropart. Phys. 87 (2017) 23.

[16] F. Schmidt, M. Ave, L. Cazon and A. Chou, Astropart. Phys. 29 (2008) 355.

[17] J. Matthews, Astropart. Phys. 22 (2005) 387.

[18] The Pierre Auger Collaboration, J. Cosmol. Astropart. Phys. 2019 (2019) 018.

[19] G. Puppi, H. Bridge and K. Greisen, Progress in Cosmic Ray Physics. Vol. 3. Edited by J.G. Wilson, North-Holland Publishing (1956).

[20] K. Kamata and J. Nishimura, Prog. Theor. Phys. Supp. 6 (1958) 93.

[21] M. Ave, M. Roth and A. Schulz, Astropart. Phys. 88 (2017) 46.

[22] D. Heck, J. Knapp, J. Capdevielle, G. Schatz, T. Thouw et al., Report FZKA 6019 (1998) .

[23] T. Pierog, I. Karpenko, J.M. Katzy, E. Yatsenko and K. Werner, Phys. Rev. C 92 (2015) 034906. 